\title{
AN EXTREMAL PROBLEM CONCERNING FINITE DIMENSIONAL SUBSPACES OF $C[a, b]$ PERTINENT IN SIGNAL THEORY
}

\author{
P. H. HALPERN ${ }^{1}$, R. N. MOHAPATRA ${ }^{2}$, P. J. O'HARA ${ }^{3}$ \\ and R. S. RODRIGUEZ ${ }^{2}$
}

(Received 21 August 1990; revised 13 March 1991)

\begin{abstract}
Increase in dimensionality of the signal space for a fixed bandwidth leads to exponential growth in the number of different signals which must be encoded. In this paper we determine the best subspace of orthogonal functions which can be used to minimise the worst ratio of peak power to RMS power. A mathematical formulation of this problem has been made and it has been found that the Fourier basis satisfies the required constraints of optimality in terms of form factor (peak/RMS ratio).
\end{abstract}

\section{Introduction}

Let $C[a, b]$ denote the collection of real-valued functions each continuous on $[a, b]$ and let $w$ be a suitable weight function, e.g., $w$ is nonnegative and integrable on $[a, b]$ and such that $\int_{c}^{d} w(x) d x>0$ for each interval $[c, d]$ contained in $[a, b]$ with $c \neq d$. Define the inner product

$$
(f, g)=\int_{a}^{b} w(x) f(x) g(x) d x, \quad f, g \in C[a, b],
$$

and consider $C[a, b]$ to be a real inner product space. For $n=1,2, \ldots$, let $\mathscr{S}_{n}$ denote the collection of all $n$-dimensional subspaces of $C[a, b]$, and

\footnotetext{
'Central Florida Technical Services, Inc., 118 Old Hickory Court, Longwood, Florida 32750.

${ }^{2}$ Department of Mathematics, University of Central FLorida, Orlando, Florida 32816.

${ }^{3}$ Deceased.
}

(C) Copyright Australian Mathematical Society 1992, Serial-fee code 0334-2700/92 
for $f \in C[a, b]$ define the norms

$$
\begin{aligned}
\|f\|_{\infty} & =\max _{a \leq x \leq b}|f(x)|, \\
\|f\|_{2} & =\sqrt{(f, f)} .
\end{aligned}
$$

A point $x_{0} \in[a, b]$ such that $\left|f\left(x_{0}\right)\right|=\|f\|_{\infty}$ will be called a norm-point of $f$. Finally if $S \in \mathscr{S}_{n}$ let

$$
\Lambda_{s}=\sup _{\substack{f \in S \\ f \neq 0}} \frac{\|f\|_{\infty}}{\|f\|_{2}} \text { and } \lambda_{n}=\inf _{S \in \mathscr{S}_{n}} \Lambda_{S} .
$$

It is clear that $\|f\|_{2}, \Lambda_{s}$, and $\lambda_{n}$ depend on the weight function, $w$, but for simplicity our notations do not indicate this dependency. The purpose of this paper is to determine the value of $\lambda_{n}$ and to characterise those "optimal" subspaces, $S \in \mathscr{S}_{n}$, for which $\Lambda_{S}=\lambda_{n}$.

Our interest in these optimal subspaces originated with an important problem in an area of application known as signal design. For our purposes a signal is a function $f$ in $C[a, b]$ where $[a, b]$ is the time interval or duration of the signal. A signal space is a finite-dimensional subspace of $C[a, b]$. When $w(x) \equiv 1$ the quantity $\|f\|_{2}$ is a measure of the energy of the signal and $\|f\|_{\infty}$ is referred to as the peak value of the signal. Due to hardware constraints it is desirable to design signal spaces which, for a given signal energy, will make the peak values of the signals as small as possible. Therefore those signal spaces which are optimal in the sense described above are of particular interest. These optimal signal spaces are also important for other considerations as mentioned in the next section.

\section{Background}

The concept of dimensionality in signal design stems from the idea that in any system with bandwidth $\beta$ there are $2 \beta \tau$ independent number of coordinates which completely describe the signal every $\tau$ seconds (see [2]). For a given average power level $S$ of a signal, Shannon [7] has proved that it is possible to transmit error free at a rate $c=\beta \log _{2}\left(1+S / N_{0}\right)$ bits, where $N_{0}$ is the noise power. However, to achieve this rate of transmission, one has to work in infinite dimensional signal space. Halpern and Mallory in their patent (see [3]) have shown that there are practical signal designs which achieve coding gain in modest dimensional spaces.

It can be observed that, with increase in dimensionality of the signal space for a fixed bandwidth, two phenomena take place:

(i) The number of different signals which must be encoded grows exponentially. 
(ii) The sphere hardening phenomenon (see [6]) occurs.

Those familiar with signal transmission know that the coding gain (i.e., increase in minimum energy of the difference signals) that we obtain with our signal design is independent of the actual subspace we use, but depends only on the geometry. However, there is one important practical problem that leads to an interesting mathematical formulation and serves as a guide to our signal designs, and that is when the transmitter is not only average-power limited but also peak-power limited.

The key question we ask is, what is the best subspace of orthogonal functions that can be used to minimise the worst ratio of peak power to RMS power? Mathematically, it amounts to the following:

For fixed $n$ and fixed time duration, find the "best" set $F$ of orthonormal functions $\left\{f_{1}, f_{2}, \ldots, f_{n}\right\}$, which minimises

$$
J_{F}=\sup _{\left\{a_{i}\right\}} \frac{\left\|\sum a_{i} f_{i}(t)\right\|}{\left(\sum\left|a_{i}\right|^{2}\right)^{1 / 2}},
$$

where $\|\cdot\|$ above is the supremum norm. Note that the problem is meaningful for real or complex $\left\{a_{i}\right\}$.

With this end in mind, we shall prove our main result in Section 3.

\section{Main Results}

The important results in this paper are corollaries of a single theorem whose proof is elementary. Before stating this theorem we introduce additional notation. If $S \in \mathscr{S}_{n}$ and $\left\{f_{1}, \ldots, f_{n}\right\}$ is an orthonormal basis for $S$ let

$$
M_{S}=\left\|\sum_{k=1}^{n} f_{k}^{2}\right\|_{\infty} .
$$

This notation might seem inappropriate, since it appears that $M_{S}$ depends not only on $S$ but also on the choice of the orthonormal basis for $S$. However, it can be shown that if $\left\{f_{1}, \ldots, f_{n}\right\}$ and $\left\{g_{1}, \ldots, g_{n}\right\}$ are both orthonormal bases for $S$, then $\sum_{k=1}^{n} f_{k}^{2}=\sum_{k=1}^{n} g_{k}^{2}$. To see this, write $g_{k}=\sum_{j=1}^{n} C_{k j} f_{j}, k=1, \ldots, n$. It is easy to verify that the $n \times n$ matrix $\left[C_{k j}\right]$ is orthogonal. A straightforward computation then shows that $\sum_{k=1}^{n} f_{k}^{2}=\sum_{k=1}^{n} g_{k}^{2}$. We now state the key theorem. 
THEOREM 1. If $S \in \mathscr{S}_{n}$ then $\Lambda_{S}=\sqrt{M}_{S}$. Moreover, the supremum $\Lambda_{S}$ is attained if and only if $f \in \mathscr{S}$ has the form

$$
f=C \sum_{k=1}^{n} \widehat{C}_{k} f_{k}, \quad C \neq 0,
$$

where $\left\{f_{1}, \ldots, f_{n}\right\}$ is any orthonormal basis for $S, \widehat{C}_{k}=f_{k}\left(x_{0}\right)$, and $x_{0}$ is any norm-point of $\sum_{k=1}^{n} f_{k}^{2}$.

Proof. Let $\left\{f_{1}, \ldots, f_{n}\right\}$ be any orthonormal basis for $S$. It is clear that

$$
\Lambda_{S}=\sup _{C_{1}, \ldots, C_{n}} \frac{\left\|\sum_{k=1}^{n} C_{k} f_{k}\right\|_{\infty}}{\left[\sum_{k=1}^{n} C_{k}^{2}\right]^{1 / 2}} .
$$

Given any choice of $C_{1}, \ldots, C_{n}$ (not all 0 ) let $x_{1}$ be a norm-point of $\sum_{k=1}^{n} C_{k} f_{k}$. Using the Cauchy-Schwarz inequality we obtain

$$
\left\|\sum_{k=1}^{n} C_{k} f_{k}\right\|_{\infty}=\left|\sum_{k=1}^{n} C_{k} f_{k}\left(x_{1}\right)\right| \leq\left[\sum_{k=1}^{n} C_{k}^{2}\right]^{\frac{1}{2}}\left[\sum_{k=1}^{n} f_{k}^{2}\left(x_{1}\right)\right]^{\frac{1}{2}} \leq\left[\sum_{k=1}^{n} C_{k}^{2}\right]^{\frac{1}{2}} \sqrt{M}_{s} .
$$

Therefore $\Lambda_{S} \leq \sqrt{M}_{S}$. Now let $x_{0}$ be a norm-point of $\sum_{k=1}^{n} f_{k}^{2}$ and let $\widehat{C}_{k}=f_{k}\left(x_{0}\right), k=1, \ldots, n$. Then

$$
\sqrt{M}_{S}=\left[\sum_{k=1}^{n} f_{k}^{2}\left(x_{0}\right)\right]^{\frac{1}{2}}=\frac{\sum_{k=1}^{n} f_{k}^{2}\left(x_{0}\right)}{\left[\sum_{k=1}^{n} f_{k}^{2}\left(x_{0}\right)\right]^{\frac{1}{2}}}=\frac{\sum_{k=1}^{n} \widehat{C}_{k} f_{k}\left(x_{0}\right)}{\left[\sum_{k=1}^{n} \widehat{C}_{k}^{2}\right]^{\frac{1}{2}}} \leq \frac{\left\|\sum_{k=1}^{n} \widehat{C}_{k} f_{k}\right\|_{\infty}}{\left[\sum_{k=1}^{n} \widehat{C}_{k}^{2}\right]^{\frac{1}{2}}} \leq \Lambda_{S} .
$$

Therefore $\Lambda_{S}=\sqrt{M}_{S}$. Moreover this implies that the last inequality in (3) is an equality and so the supremum, $\Lambda_{S}$, is attained when $f$ is of the form described by (1). Conversely, suppose $f=\sum_{k=1}^{n} C_{k} f_{k}$ is such that $\|f\|_{\infty} /\|f\|_{2}=\Lambda_{S}$ and let $x_{1}$ be a norm-point of $f$. Then the inequalities in (2) would be equalities, from which it would follow that $x_{1}$ is a norm-point of $\sum_{k=1}^{n} f_{k}^{2}$ and $C_{k}=C f_{k}\left(x_{1}\right), k=1, \ldots, n$, for some $C \neq 0$.

Corollary 1. For $n=1,2, \ldots$

$$
\lambda_{n}=\left[n /\left(\int_{a}^{b} w(x) d x\right)\right]^{1 / 2} .
$$


Moreover a subspace $S \in \mathscr{S}_{n}$ is optimal (i..e, $\Lambda_{S}=\lambda_{n}$ ) if and only if $\sum_{k=1}^{n} f_{k}^{2}$ is a constant function whenever $\left\{f_{1}, \ldots, f_{n}\right\}$ is an orthonormal basis for $S$.

Proof. If $S \in \mathscr{S}_{n}$ and $\left(f_{1}, \ldots, f_{n}\right\}$ is an orthonormal basis for $S$ then

$$
\begin{aligned}
n=\sum_{k=1}^{n}\left[\int_{a}^{b} w(x) f_{k}^{2}(x) d x\right] & =\int_{a}^{b}\left[w(x) \sum_{k=1}^{n} f_{k}^{2}(x)\right] d x \\
& \leq M_{S} \int_{a}^{b} w(x) d x=\Lambda_{S}^{2} \int_{a}^{b} w(x) d x .
\end{aligned}
$$

Moreover, the inequality above is equality if and only if $\sum_{k=1}^{n} f_{k}^{2}$ is a constant. Therefore the proof will be complete once we show the existence of an orthonormal set $\left\{f_{1}, \ldots, f_{n}\right\}$ for which $\sum_{k=1}^{n} f_{k}^{2}$ is a constant. For this purpose, let $W(x)=A \int_{a}^{x} w(t) d t$ where $A=2 \pi /\left[\int_{a}^{b} w(t) d t\right]$, and let $k_{1}, \ldots, k_{m}$ be $m$ distinct positive integers. Then when $n=2 m$, $\left\{(A / \pi)^{1 / 2} \sin k_{i} W(x),(A / \pi)^{1 / 2} \cos k_{i} W(x)\right\}_{i=1}^{m}$ is an orthonormal set of $n$ functions whose sum of squares is constant. Adding the constant function $(A / 2 \pi)^{1 / 2}$ to this set handles the case when $n=2 m+1$.

\section{Remarks}

It is worth noting the following "pointwise" version of Theorem 1, whose proof is also an immediate consequence of the Cauchy-Schwarz inequality.

Theorem 2. If $S \in \mathscr{S}_{n}$ and $\left\{f_{1}, \ldots, f_{n}\right\}$ is any orthonormal basis for $S$ then for any $x_{0} \in[a, b]$,

$$
\sup _{\substack{f \in S \\ f \neq 0}} \frac{\left|f\left(x_{0}\right)\right|}{\|f\|_{2}}=\left[\sum_{k=1}^{n} f_{k}^{2}\left(x_{0}\right)\right]^{1 / 2} .
$$

Moreover, if we assume $\sum_{k=1}^{n} f_{k}^{2}\left(x_{0}\right)>0$, then this supremum is attained if and only if $f \in S$ has the form

$$
f=C \sum_{k=1}^{n} f_{k}\left(x_{0}\right) f_{k}, \quad \text { for some } C \neq 0 .
$$

By applying Theorems 1 and 2 to the case where $S$ is the subspace of real algebraic polynomials of degree $\leq n-1,[a, b]=[-1,1]$ and $w$ is one of the weights defined by $1,1 / \sqrt{1-x^{2}}$, or $\sqrt{1-x^{2}}$ we obtain the following results, some of which can be found in [5], pp. 89-90. Let $P_{k}, T_{k}$, 
$U_{k}, k=0,1,2, \ldots$, denote respectively the Legendre and Tschebysheff polynomials of the first and second kind (see [1]).

COROLlaRY 2. If $p$ is any real algebraic polynomial of degree $\leq n-1$ and $x_{0} \in[-1,1]$ then

$$
\begin{gathered}
\max _{-1 \leq x \leq 1}|p(x)| \leq \frac{n}{\sqrt{2}}\left[\int_{-1}^{1} p^{2}(x) d x\right]^{1 / 2} \\
\left|p\left(x_{0}\right)\right| \leq\left[\sum_{k=0}^{n-1}\left(\frac{2 k+1}{2}\right) P_{k}^{2}\left(x_{0}\right)\right]^{1 / 2}\left[\int_{-1}^{1} p^{2}(x) d x\right]^{1 / 2} \\
\max _{-1 \leq x \leq 1}|p(x)| \leq\left[\frac{2 n-1}{\pi}\right]^{1 / 2}\left[\int_{-1}^{1} \frac{p^{2}(x)}{\sqrt{1-x^{2}}} d x\right]^{1 / 2} \\
\left|p\left(x_{0}\right)\right| \leq\left[\frac{1}{\pi}+\frac{2}{\pi} \sum_{k=1}^{n-1} T_{k}^{2}\left(x_{0}\right)\right]^{1 / 2}\left[\int_{-1}^{1} \frac{p^{2}(x)}{\sqrt{1-x^{2}}} d x\right]^{1 / 2} \\
\max _{-1 \leq x \leq 1}|p(x)| \leq\left[\frac{n(n+1)(2 n+1)}{3 \pi}\right]^{1 / 2}\left[\int_{-1}^{1} p^{2}(x) \sqrt{1-x^{2}} d x\right]^{1 / 2} \\
\left|p\left(x_{0}\right)\right| \leq\left[\frac{2}{\pi} \sum_{k=0}^{n-1} U_{k}^{2}\left(x_{0}\right)\right]^{1 / 2}\left[\int_{-1}^{1} p^{2}(x) \sqrt{1-x^{2}} d x\right]^{1 / 2}
\end{gathered}
$$

For each of the inequalities in the above corollary, necessary and sufficient conditions for equality can be deduced from Theorems 1 and 2. For example, there is equality in (4) if and only if

$$
p=C \sum_{k=0}^{n-1}(2 k+1) P_{k} \quad \text { or } \quad p=C \sum_{k=0}^{n-1}(-1)^{k}(2 k+1) P_{k}, \quad \text { for some } C \neq 0 .
$$

We note also that Theorem 2 holds when $[a, b]$ is an infinite interval provided the weight, $w$, and the subspace $S$, are chosen appropriately, i.e., $\int_{a}^{b} w(x) f(x) g(x) d x$ is a real number for all $f, g \in S$; as a consequence we obtain (see [5], pp. 89-90):

COROLlaRY 3. If $p$ is any real algebraic polynomial of degree $\leq n-1$ then $\left|p\left(x_{0}\right)\right| \leq\left[\frac{1}{\sqrt{\pi}} \sum_{k=0}^{n-1} \frac{H_{k}^{2}\left(x_{0}\right)}{\left(2^{k}\right)(k !)}\right]^{1 / 2}\left[\int_{-\infty}^{\infty} p^{2}(x) e^{-x^{2}} d x\right]^{1 / 2}, \quad-\infty<x_{0}<\infty$, 
and

$$
\left|p\left(x_{0}\right)\right| \leq\left[\sum_{k=0}^{n-1} \frac{k !\left(L_{k}^{(\alpha)}\left(x_{0}\right)\right)^{2}}{\Gamma(k+\alpha+1)}\right]^{1 / 2}\left[\int_{0}^{\infty} x^{\alpha} e^{-x} p^{2}(x) d x\right]^{1 / 2}, \quad \alpha>-1, x_{0}>0 .
$$

Here $H_{k}$ and $L_{k}^{(\alpha)}, k=0,1,2, \ldots$, are the Hermite polynomials with weight $e^{-x^{2}}$ and the generalised Laguerre polynomials respectively, e.g. see [1].

As a final remark, we note that an orthonormal system whose span is an optimal space (i.e., an optimal orthonormal system) may be of interest, and the construction of such an optimal system (using sines and cosines) is demonstrated in the proof of Corollary 1 . The following proposition gives another method for producing optimal systems for weight 1 .

Proposition. Suppose

(i) $a>0, f \in C[0, a], \int_{0}^{a} f^{2}(x) d x=\frac{1}{2}, \max _{0 \leq x \leq a}|f(x)|=f(0)=\frac{1}{\sqrt{a}}$ and $f(a-x)=-f(x)$ for $x \in[0, a]$

(ii) $g(x)=\left[f^{2}(0)-f^{2}(x)\right]^{1 / 2}$ for $x \in[0, a]$;

(iii) $F$ is the even periodic extension with period $2 a$ of $f$ to $\mathscr{R}=(-\infty, \infty)$, e.g., require $f(x)=f(-x)$ for $x \in[-a, 0]$ and then $f(x+2 a)=$ $f(x)$ each $x$

(iv) $G$ is the odd periodic extension with period $2 a$ of $g$ to $\mathscr{R}$. Then the system $(2 a)^{1 / 2}, F(x), G(x), F(2 x), G(2 x), \ldots, F\left(2^{l} x\right), G\left(2^{l} x\right)$ is an optimal orthonormal system on $[-a, a], l=0,1,2, \ldots$ Moreover the system with the constant term deleted is also an optimal orthonormal system.

For a proof of the proposition, one verifies that $F$ and $G$ defined by (iii) and (iv) are continuous functions on $(-\infty, \infty)$, have period $2 a$, and satisfy

$$
(F(x))^{2}+(G(x))^{2}=(F(0))^{2}
$$

for each $x$. Then, by using induction and properties of $F$ and $G$, one verifies that the system of functions (with or without the constant term) is orthonormal on $[-a, a]$. The conclusion that the system of functions is an optimal orthonormal system on $[-a, a]$ follows from Corollary 1 .

Conclusion In this paper we have considered a problem concerning channel coding by characterising finite dimensional signal spaces of orthogonal functions which minimise the worst ratio of peak power to RMS power. It has been shown that the Fourier basis satisfies the required constraints of optimality in terms of the form factor(peak/RMS ratio). In the last proposition, 
a method of obtaining other optimal systems is outlined. Based on our result, one can conclude that the alphabets of signals should use a chosen subset of harmonics.

We thank the referee for pointing out that our construction of optimal functions in the proposition may be related to the construction of orthonormal wavelet bases considered by I. Daubechies, "Orthonormal bases of compactly supported wavelets," Comm. Pure Appl. Math. 41 (1988), 909-986.

\section{References}

[1] P. J. Davis, Interpolation and approximation, (Dover Publications, New York, 1975).

[2] Lewis Franks, Signal theory, (Prentice-Hall, 1969).

[3] Peter H. Halpern and Peter E. Mallory, "Method and apparatus for transmitting data over limited bandwidth channels," United States Patent \#4, 403, 331 (1983).

[4] A. Papoulus, The Fourier integral and its applications, (McGraw-Hill, 1969).

[5] G. Pólya and G. Szegö, Problems and theorems in analysis (Vol. II), (Springer-Verlag, 1976).

[6] C. E. Shannon, "Certain results in coding theory for noisy channels," Journal of Information and Control (1957).

[7] C. E. Shannon, "Probability of error for optimal codes in a Gaussian channel," Bell Systems Tech. Journal 38 (1959) 611-656. 